\title{
Study of the Crosslinking of PVA with Glyoxal in LbL Nanocomposites
}

\author{
Fatma Ben Dhieb', Adrián Carrillo García², Seyed H. Tabatabaei ${ }^{3}$ Frej Mighri, Abdellah Ajji ${ }^{*}$ \\ ${ }^{1}$ Chemical Engineering Department, Polytechnique Montreal, CREPEC, Montreal, Canada \\ ${ }^{2}$ Chemical Engineering Department, Polytechnique Montreal, Montreal, Canada \\ ${ }^{3}$ ProAmpac, Terrebonne, Canada \\ ${ }^{4}$ Chemical Engineering Department, Laval University, CREPEC, Quebec City, Canada \\ Email: *abdellah.ajji@polymtl.ca
}

How to cite this paper: Dhieb, F.B., García, A.C., Tabatabaei, S.H., Mighri, F. and Ajji, A. (2019) Study of the Crosslinking of PVA with Glyoxal in LbL Nanocomposites. Open Journal of Polymer Chemistry, 9, 100-116.

https://doi.org/10.4236/ojpchem.2019.94009

Received: July 22, 2019

Accepted: October 29, 2019

Published: November 1, 2019

Copyright (c) 2019 by author(s) and Scientific Research Publishing Inc. This work is licensed under the Creative Commons Attribution International License (CC BY 4.0).

http://creativecommons.org/licenses/by/4.0/

\begin{abstract}
Crosslinking is a common practice to improve the barrier properties of polymers. In this study, Montmorillonite (MMT) was used with Polyvinyl alcohol (PVA) to deposit nanocomposite coatings which were crosslinked with glyoxal (Gly) by Layer by Layer (LbL) on a PET substrate. Two crosslinking conditions were studied, under mild condition and with an acidic environment. Mild condition was useful to identify the reversibility steps and the optimum crosslinking times while the acidic environment was essential to investigate the crosslinking mechanism, by determining the permeability for different crosslinking times. PVA and PVA-MMT coatings showed a strong correlation between the permeability coefficients for different crosslinking times and the FTIR results.
\end{abstract}

\section{Keywords}

Glyoxal, LbL, Nanocomposite, PVA, Crosslinking Mechanism

\section{Introduction}

Barrier materials are in constant demand for a wide variety of applications, especially for food packaging which has a considerable growth of the flexible packaging market. Polymeric materials are a considerable part of this market and many technologies are currently applied to improve their gas barrier. The most popular, is the multilayer structured film in which a barrier layer (ethylene vinyl alcohol (EVOH), Nylon...) is usually part of a three to twelve layers structure. Metallic and silicon oxide coatings are also extensively used for high barrier packaging. In addition to these two, other techniques are also used to improve the cost and 
the recyclability like polymer blending, and the addition of fillers.

Nanocomposite coatings are considered a sustainable solution, due to their recyclability and the increasing interest in ecofriendly materials like clay [1], cellulose nanocrystals [2] or chitosan [3].

For nanocomposites, some affordable materials like clay, improves considerably the polymer properties even with adding low amounts, due to its high aspect ratio [4]. The dispersion and exfoliation of clay depend mainly on its interaction with the polymer and the applied process. Especially for melting and casting, high clay loading can't be reached due to its agglomeration that may deteriorate the barrier properties [5]. Coating, in the other hand, resulted in a better clay dispersion and intercalation [6].

Among the coating techniques, Layer by Layer (LbL) was developed in the early 90's and consists in the alternate deposition of polymer layers [7]. The filler can be added whether directly to the polymer solution that will be deposited, or it can be deposited as a separate layer by dipping (for dip coating) the substrate in the filler solution. LbL has shown interesting results compared to conventional techniques by reducing oxygen permeability by up to $99.9 \%$ [8]. Obtained coatings have a tailored thickness that depends on the number of layers, the properties of the used materials (fillers and polymers) and the coating steps. To increase gas barrier and reduce the sensitivity to moisture, these coatings are usually chemically crosslinked [9]. For polymers with hydroxyl and amine functional groups, dialdehydes in particular are widely used. Their remarkable reactivity results in a high crosslinking density, reducing thus considerably the free volume in the film. Other than the gas barrier, crosslinking improves also the mechanical properties [10] due to the modification of the polymer structure with the established covalent bonds [11]. The acetal formation and crosslinking reaction is known to be reversible, which could be avoided with acidic conditions. Some studies conducted this reaction under mild conditions and resulted in a non proportionality between the crosslinker concentration and the density of crosslinking [12]. Most of the crosslinking studies consider a homogeneous system in which the crosslinker solution is added to the polymer solution [11] [13] while heterogeneous reaction is not extensively studied.

To the best of our knowledge, this is the first thorough study of the variation of permeability with crosslinking time, the tracking of the acetal reaction reversibility and the correlation of permeability and FTIR absorption intensity for LbL coatings.

In this study crosslinking mechanism of a nanocomposite coating by glyoxal was investigated by comparing the impact of crosslinking time on the permeability of PVA-MMT and PVA coatings. To investigate the steps of a reversible reaction, both coatings were also crosslinked without the presence of an acid. The bonding variation in PVA based coatings was explored by FTIR characterization and a correlation was deduced between the permeability and the intensity of FTIR peaks. The hydrophobicity of their surface was also determined by water 
contact angle measurement.

\section{Experimental}

\subsection{Materials}

PVA $\left(\mathrm{Mw}=140,000-186,000 \mathrm{~g} \cdot \mathrm{mol}^{-1}\right)$ was purchased from Sigma Aldrich (Saint Louis, Missouri, USA) and Natural sodium montmorillonite (MMT) (Cloisite $\mathrm{NA}^{+}$) with a density of $2.86 \mathrm{~g} / \mathrm{cm}^{3}$, supplied by BYK (Gonzales, Texas, United States), was used as received. Silicon wafers, purchased from EL-Cat Inc. (Ridgefield Park, New Jersey, United States) were also used as substrate. They were used for the FTIR characterization due to their transparency to infra-red. The selected substrate for permeability tests and the other characterizations, is a polyethylene terephthalate (PET) film, $16 \mu \mathrm{m}$, provided by ProAmpac (Terrebonne, QC, Canada). The used primer is a polyethylene imine solution (PEI) (5\% aqueous solution), purchased from MICA Corporation (Shelton, CT, USA). Glyoxal, the crosslinker, (40\% aqueous solution) and Hydrochloric acid (37\%) were purchased from Sigma Aldrich (Saint Louis, Missouri, USA).

\subsection{Dip Coating Preparation}

PVA solution was prepared with deionized (DI) water with continuous stirring for $2 \mathrm{~h}$ and heating at $80^{\circ} \mathrm{C}$. MMT suspension in DI was stirred for at least 4 hours. Deposition of a coating by LbL is a repetition of three steps to deposit each layer: dipping of the substrate in the PVA or MMT solution, rinsing and drying. For the first bilayer (the first layers of PVA and MMT) the time for each step is higher to have a better coverage. Crosslinking the LbL coatings consists in dipping the coated substrate in the glyoxal solution (5\%), twice: after the deposition of half the layers and at the end when all the bilayers are deposited. For crosslinking reaction with the presence of acid, hydrochloric acid $(0.1 \mathrm{M})$ is added to the crosslinker solution.

\subsection{Determination of the Solubility and Diffusion Coefficients}

To determine the solubility and diffusion coefficients of the coated substrate, the unsteady state is required. To achieve it, the tested films are first conditioned in the MOCON apparatus with nitrogen only for several hours, and then tested with nitrogen in order to remove all the oxygen trapped in the film. A test of the oxygen permeability is started directly afterwards without conditioning. With this unsteady state, Fick's second law can be applied, Equation (1). Diffusion and permeability coefficients are determined with the solution to this law, Equation (2), and the solubility is calculated with Equation (3).

$$
\begin{gathered}
\frac{\partial C}{\partial t}=D \frac{\partial^{2} C}{\partial x^{2}} \\
J(t)=\frac{P p}{l}\left[1+2 \sum_{n=1}^{\infty}(-1)^{n} \exp \left(-\frac{D \pi^{2} n^{2} t}{l^{2}}\right)\right]
\end{gathered}
$$




$$
P=S \cdot D
$$

\section{Characterization Techniques}

The interlayer spacing d (001) of MMT was determined according to Bragg's law, by using X-ray diffraction spectroscopy (Philips X'pert) in the wide-angle mode and copper CuKa radiation $(\lambda=1.54056 \AA)$ as a source. The coatings were scanned with a rate of $0.02 \%$ in the range of 2 to 10 degrees. MOCON OXTRAN 2/21 (Minneapolis, USA) apparatus was used to measure the permeability to oxygen at $0 \%$ relative humidity, $25^{\circ} \mathrm{C}$, and $1 \mathrm{~atm}$ pressure, as described in the ASTM standard, D-3985-81. For a higher relative humidity (70\%), tests were conducted with a MOCON OXTRAN 10X apparatus (Minneapolis, USA), at $25^{\circ} \mathrm{C}$.

The contact angle of the coated PET was determined with the sessile drop method by using a Goniometer OCA20 from dataphysics. Measures were done at room temperature and distilled water was the probe liquid. To determine the contact angle, the drop image to analyze is considered the one taken after 5 seconds from the deposition of the drop on the surface. The reported values are an average of five readings at different location of the sample's surface. Coatings thickness was measured with a Dektak 3030 stylus profilometer with silicon plates as substrate. Transparent to Infrared silicon plates constituted the substrate for FTIR testing. Coatings were scanned in transmission mode in the range 600 to $4000 \mathrm{~cm}^{-1}$ with a resolution of $4 \mathrm{~cm}^{-1}$. The spectrum of the silicon substrate was subtracted afterwards from the analyzed spectra. To determine the percentage per weight of clay, thermogravimetric analysis was conducted with a TGA Q500 from TA instruments, by heating the sample up to $800^{\circ} \mathrm{C}$. At this temperature, the residual weight is the one of clay only.

\section{Results and Discussion}

\subsection{Crosslinking Reaction of Hydroxyls with Glyoxal in a Homogeneous System}

Polymer crosslinking studies focused on extrusion and solution casting by adding the crosslinker solution to the melt or polymer solution, which are homogeneous reactions. Nonetheless, those studies differ from LbL coatings where the crosslinking is performed by the addition of the crosslinker solution to a LbL deposited film which was previously dried. As such, the crosslinking can be assumed as a heterogeneous reaction.

The reaction mechanism for some crosslinkers, e.g. glyoxal, can vary depending on the polymer functional group. For instance, for aldehydes crosslinkers the crosslinking mechanism with polymers containing hydroxyl groups in their structure, e.g. PVA, is based on two reactions occurring in an acidic environment. In the case of PVA, presented in Figure 1, it first reacts with Gly to result in an unstable intermediate, the hemi acetal, which then reacts with the PVA to form the acetal and water. Both reactions are reversible in the absence of acid. 


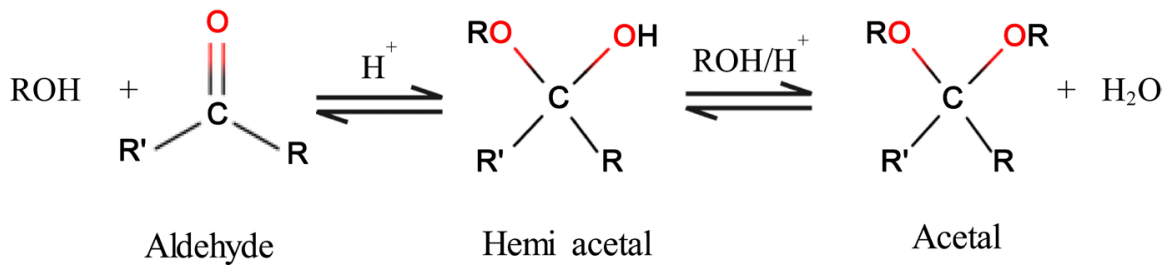

Figure 1. Crosslinking reaction of hydroxyl groups with dialdehydes.

The kinetic study of the crosslinking of aliphatic alcohol with aldehydes to form acetals, for homogeneous systems, is presented as a second order reaction with two reactants (Equation (4)) [14]:

$$
-\frac{\mathrm{d}[\mathrm{Gly}]}{\mathrm{d} t}=k \cdot[\mathrm{Gly}] \cdot[\mathrm{PVA}]
$$

According to a study conducted by Kormanovskaya et al. (1964), with PVA and formaldehyde, the second reaction involving the reaction of hemiacetal with PVA is the limiting step of the crosslinking reaction [15]. To make the reaction run in the forward direction, avoiding the reversibility of the crosslinking mechanism, the use of high initial concentration of PVA was suggested.

\subsection{Mild Conditions for PVA Crosslinking}

Most of the studies of the crosslinking of PVA with aldehydes consider a homogeneous system, in which the crosslinker solution is added to the PVA solution. This is not the case for LbL deposited film, as whether for spin, spray or dip coating, the crosslinker solution is applied to a dry film. For dipping, for instance, the LbL film is crosslinked by dipping it in the crosslinker solution. Crosslinking LbL deposited films is a heterogeneous reaction that implies a crosslinker diffusion with a simultaneous reaction.

The presence of acid in the acetal reaction is important for limiting the reversibility of both reaction steps, by pushing the reaction toward the formation of acetal (Figure 2).

The addition of acid can, however, be considered aggressive for some applications. Applying mild conditions by using the crosslinker only without acid, yields to a reversible reaction. The steps of this reversibility can be clearly identified when measuring the permeability of the coatings for different crosslinking times (Figure 2). This is an interesting observation as it allows to investigate the effect of the crosslinking on the reaction reversibility.

The crosslinking reaction was studied for up to $60 \mathrm{~min}$ of reaction time. For the first 45 minutes of crosslinking there's two increases in the permeability at 15 and $30 \mathrm{~min}$. For the first reached maximum of $P / P_{0}$, the value 0.6 is obtained whereas for the second, this value is lowered to 0.2 . This can be explained by the fact that it's a reversible two steps reaction.

For the first step, one hydroxyl group reacts and for the second, both are linked to the polymer chain. The free volume is then different in both steps and induces a lower permeability for the acetal state. The permeability in function of 


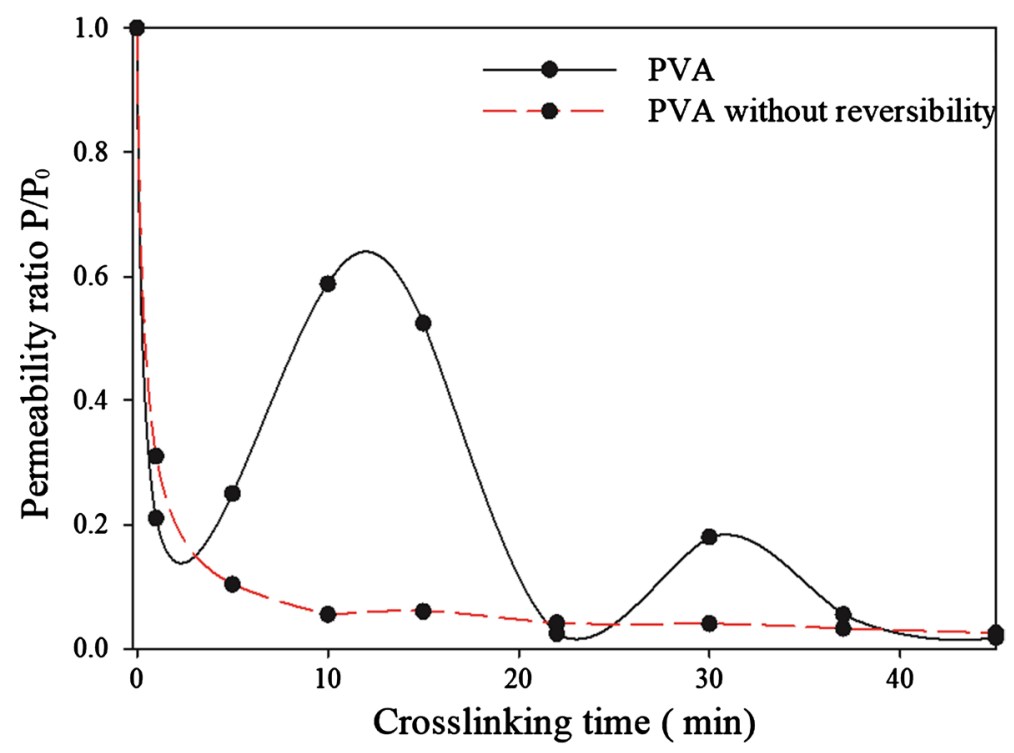

Figure 2. LbL assembly of PVA layers with and without acidic environment.

the crosslinking time curve shows clearly the different crosslinking stages.

The two reached maxima are around $10-15 \mathrm{~min}$ and $30 \mathrm{~min}$ with a permeability reduced three times for the second maximum compared to the first. The lowest permeabilities follow also the same trend as the highest, with first a minimum around $0.2 \mathrm{cc} / \mathrm{m}^{2} \cdot$ day.atm during the first $5 \mathrm{~min}$ and a second minimum with half the permeability of the first $\left(0.1 \mathrm{cc} / \mathrm{m}^{2} \cdot\right.$ day.atm $)$ for $22 \mathrm{~min}$ crosslinking time. This reversibility is attenuated with longer crosslinking time, as permeability is lower than $0.06 \mathrm{cc} / \mathrm{m}^{2}$.day.atm for times higher than $37 \mathrm{~min}$ utes. It's clear that beyond this time, the maximum reduction of free volume is achieved meaning the predominance of acetals in the film. Knowing that the possible states of crosslinked Gly are hemiacetal or acetal and that Gly may establish four covalent bonds or less, such gradual reduction in permeability may be related to the steps of the reversible crosslinking reaction. The 0.2 value is reached twice, between 1 and $5 \mathrm{~min}$ as a minimum and at $30 \mathrm{~min}$ as a maximum. As an intermediate value, this would correspond to a predominance of the hemi acetal state. This would also be explained by the fact that's in between two maxima in the first case and two minima in the second. The two maxima correspond to the non crosslinked state and a partly crosslinked state with hemi acetal whereas the two minima are due to the predominance of acetals in the coating. Even though, this reversibility has a more attenuated effect on the permeability with longer crosslinking time, with the presence of minima around 5 and 20 minutes, choosing the right crosslinking time can yield a high barrier film without the need to use an acidic environment or very long crosslinking times

\subsection{Effect of Fillers in the Diffusion of the Crosslinker}

The nanocomposite PVA-MMT film has an intra and interchain hydrogen bonding as well as a bonding with the MMT. With the crosslinker, there is break- 
age of those bonds and a creation of a more solid and short ones, the covalent bonds. During this reversible reaction, there's a constant variation in the bonds type and as the length and intensity of these bonds is different, it contributes differently to the reduction of free volume in the coating. Hydrogen bonds between hydroxyl groups have a higher length than $\mathrm{C}-\mathrm{O}$ covalent bond $(1.85 \AA$ [16] compared to $1.44 \AA$ for a covalent bond).

As PVA-MMT assembly is based on hydrogen bonding and this affinity affects the clay properties, the interlayer spacing of MMT was determined for several crosslinking times and the result showed that crosslinking didn't affect considerably this spacing (Figure S1).

MMT increases the tortuosity in the coating by increasing the path of diffusing molecules but it also affects the mobility of PVA chains [17], which may hinder the diffusion of Gly, considering that MMT represents 60 wt $\%$ of the coating (Figure S2). Plotting relative permeability in function of the crosslinking time for both coatings, with and without MMT (Figure 3), showed a better reduction in permeability for PVA coatings. Both types of coatings had a similar variation of $P / P_{0}$ with the crosslinking time, meaning that MMT didn't affect the different steps of the reaction and its reversibility. The only difference observed is the lower permeability ratio of PVA coating for the first $30 \mathrm{~min}$ of crosslinking. Gly molecules diffuse through the coating while reacting with hydroxyl groups to establish covalent bonds which could slow the reaction rate, such assumption must be confirmed by a kinetic study.

The lower permeability coefficients for nanocomposites compared to the neat polymers is often interpreted with the reduction of the diffusion of permeant gas molecules, making the free volume an important nanocomposite property to be considered. This latter has been described as having an exponential effect on the permeability [17].

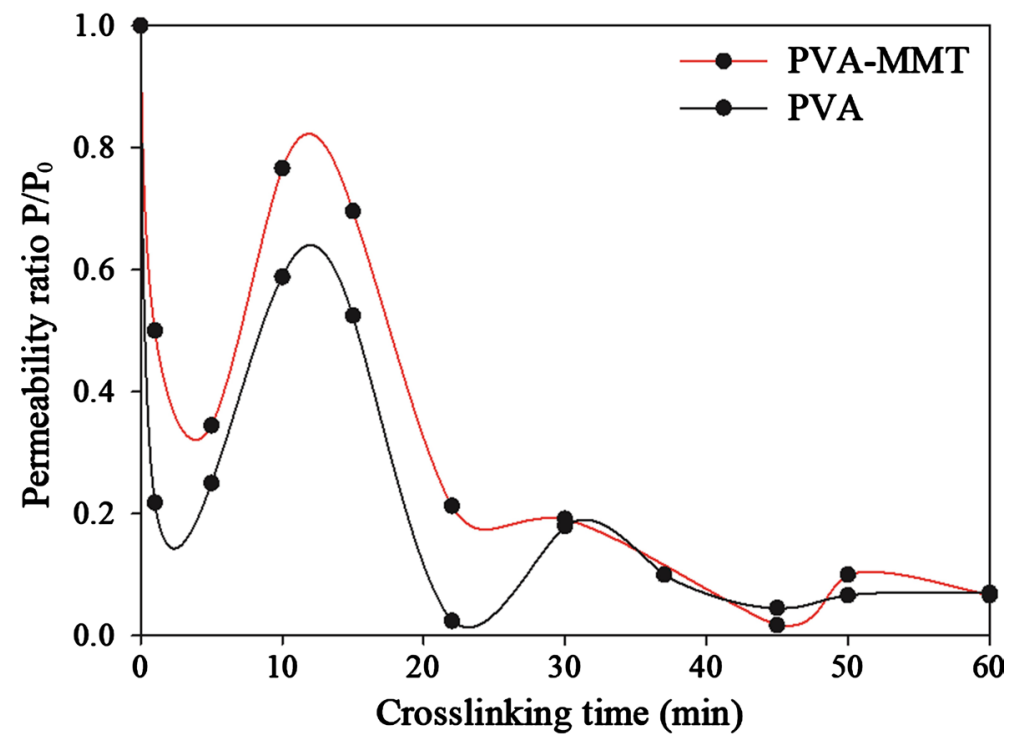

Figure 3. Permeability ratio in function of crosslinking time for LbL coated PET with PVA and PVA-MMT coatings. 
Permeability is, however, also a function of the solubility coefficient which is specific to the pair polymer-permeant molecule. Gas solubility is related to the static free volume and is independent of the polymer chains motions while its diffusivity is related to the dynamic free volume due to the motion of the polymer segments.

Diffusion coefficient is thus a kinetic factor that gives an understanding of the mobility of permeant molecules in the polymer. Solubility coefficient, in the other hand, is a thermodynamic factor that depends on the affinity between gas molecules and the polymer [18].

The presence of MMT in the LbL coating decreases both coefficients (Table 1). The diffusivity coefficient decreases around 5 times for $5 \mathrm{~min}$ crosslinked coatings, due to the higher tortuosity in the nanocomposite and the reduction of the free volume. The solubility decreases considerably with the addition of MMT with a 15 times reduction. For both PVA and PVA-MMT coatings, crosslinking did not affect diffusion and had a more pronounced impact on solubility instead. A higher reduction is obtained with a longer crosslinking time, as a $50 \mathrm{~min}$ crosslinking time resulted in a 10 times lower solubility coefficient for the PVA-MMT coating than a 5 min crosslinking time.

Contrary to the bulk, reversibility did not seem to affect the coatings surface hydrophilicity (Figure 4). The water CA varies according to the density of free

Table 1. Diffusivity and solubility coefficients for different PVA and PVA-MMT coatings.

\begin{tabular}{ccc}
\hline Coating & $D\left(\mathrm{~cm}^{2} / \mathrm{s}\right)$ & $S\left(\mathrm{~cm}^{3} / \mathrm{cm}^{3} \cdot \mathrm{atm}\right)$ \\
\hline 15 PVA, Gly 5 min & $3.18 \mathrm{e}-9$ & 10.88 \\
15 PVA & $2.4 \mathrm{e}-9$ & 50.44 \\
15 PVA MMT, Gly 5 min & $7.63 \mathrm{e}-10$ & 0.72 \\
15 PVA MMT, Gly 50 min & $8.47 \mathrm{e}-10$ & 0.079 \\
\hline
\end{tabular}

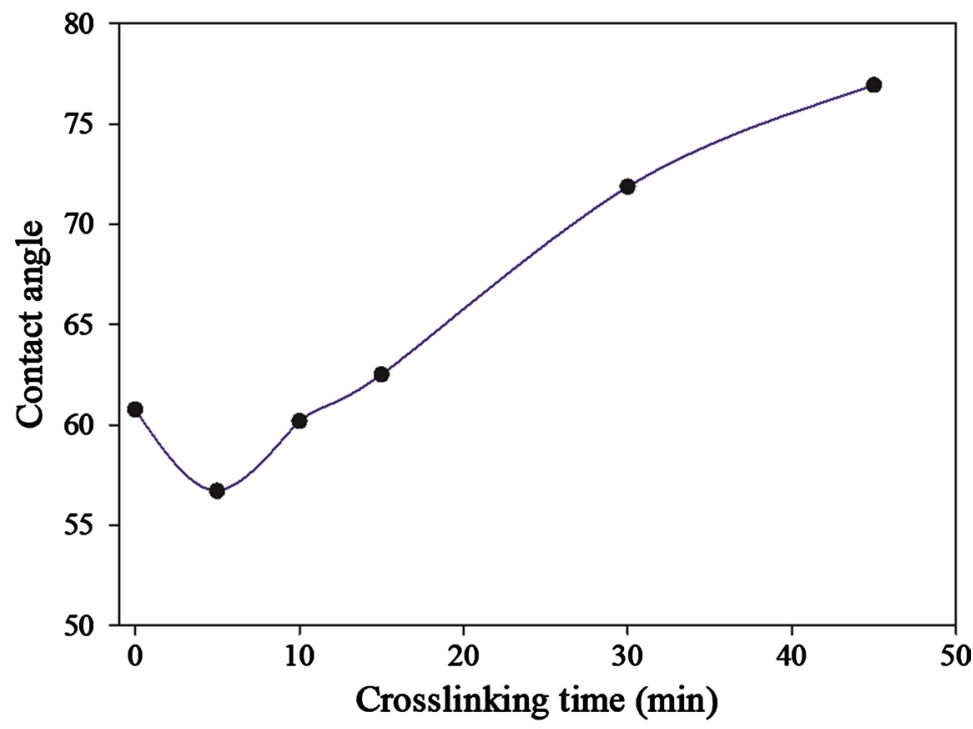

Figure 4. Water contact angle of PVA-MMT coatings at different crosslinking times. 
hydroxyl groups at the surface as they can establish hydrogen bonds with the water. The lower the CA, the higher the surface hydrophilicity. For crosslinked PVA-MMT coatings without added acid, surface hydrophobicity increased proportionally to the crosslinking time. Crosslinking for a dipping process implies a reaction/diffusion mechanism. During the crosslinking, the coating is dipped in the crosslinker solution which keeps the surface in constant contact with the crosslinker molecules. Such availability of the crosslinker may explain the increase in the surface hydrophobicity with the crosslinking time despite the reversibility of the reaction.

\subsection{Investigation of the Crosslinking Reaction}

FTIR spectroscopy is a reliable characterization technique to investigate the variation in the bonding for the studied material. The spectra of PVA and PVA-MMT coatings with different crosslinking times were collected by transmission mode (Figure 5). The peak linked to the stretching of the $\mathrm{C}-\mathrm{O}$ bond in the PVA-MMT coatings is at $1047 \mathrm{~cm}^{-1}$ whereas it is at $1076 \mathrm{~cm}^{-1}$ for PVA-coatings. This bond is usually at $1092 \mathrm{~cm}^{-1}$ for neat PVA but it shifted due to hydrogen bonding (for both coatings) and due to overlapping with MMT peak, in the case of the nanocomposite coating. As the $\mathrm{C}-\mathrm{O}$ is the established bond by crosslinking, the variation of the peak intensity reflects the extent of crosslinking. Following Beer Lambert's law, the intensity of this peak should be proportional to the concentration of the $\mathrm{C}-\mathrm{O}$ bond in the coating which means its proportionality to the crosslinking density.

For PVA coatings, a clear difference between the intensities of the C-O peak for the 10 and 15 min crosslinking times and the remaining crosslinking times is observed which corroborate their lower permeabilities. For PVA-MMT coatings, the 5 min time has an intermediate intensity between low permeability and high permeability coatings dividing them in two distinct groups. The high permeability group consists of $0,1,10$ and $15 \mathrm{~min}$ and the low permeability one includes the 30, 45, 60 and 90 min crosslinking times. The 90 min crosslinking time was only characterized by FTIR to corroborate the obtained results, and according to the obtained spectra the permeability should be lower than $0.04 \mathrm{cc} / \mathrm{m}^{2} \cdot$ day.atm.

When plotting permeability results in function of the $\mathrm{C}-\mathrm{O}$ peak intensities, for PVA and PVA-MMT coatings with and without reversibility, a linear correlation is obtained (Figure 6). Such correlation enables a different approach in interpreting the permeability results.

This linear relation between the permeability and the peak intensity means that the permeability is also linearly proportional to the crosslinked groups concentration. As the determined diffusion and solubility coefficients showed, solubility is more affected by the crosslinking than diffusivity. With such logic, solubility is thus linearly proportional to the concentration of crosslinked groups.

By establishing this correlation for the same material and coating technique, the permeability can be estimated, with only the coated samples needed for 

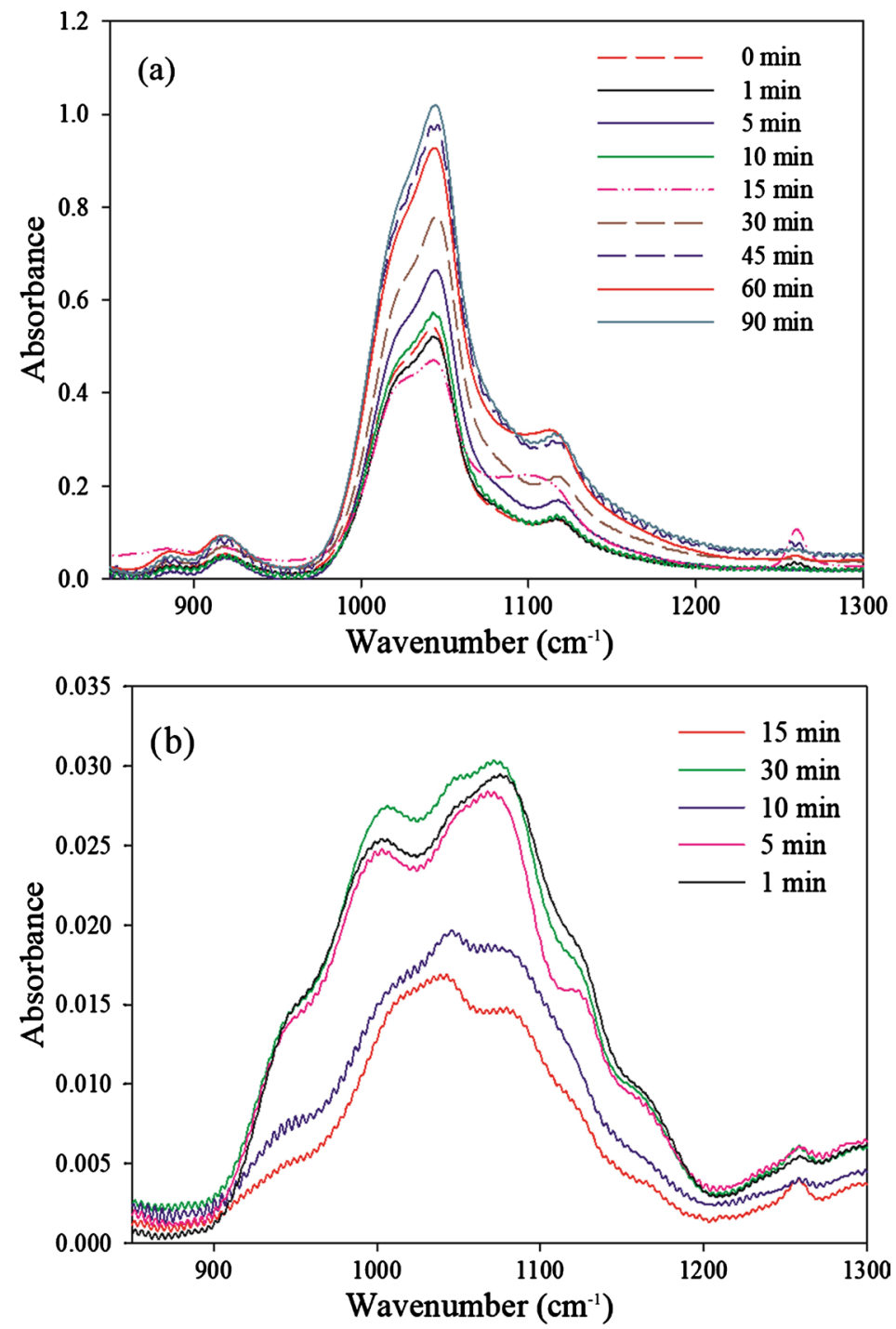

Figure 5. FTIR spectra of different crosslinking times for (a) PVA-MMT coatings and (b) PVA only coating.

FTIR, cutting thus drastically the experimental time.

\subsection{Reaction Kinetic}

For a homogeneous crosslinking reaction, the considered reaction kinetic depends on both PVA and Gly with an order of 1 for each of them. In the case of LbL, two main differences exist, compared to the usually studied homogeneous reactions. On one hand, it is a heterogeneous reaction which affects the reaction kinetic, and on the other hand, for the crosslinking of LbL films, PVA is in excess compared to the crosslinker which simplifies Equation (4) to Equation (5). It must be mentioned that it is very difficult to determine the concentrations of both the PVA and the Gly in the LbL coating.

$$
-\frac{\mathrm{d}[\mathrm{Gly}]}{\mathrm{d} t}=k \cdot[\mathrm{Gly}]^{\alpha}
$$



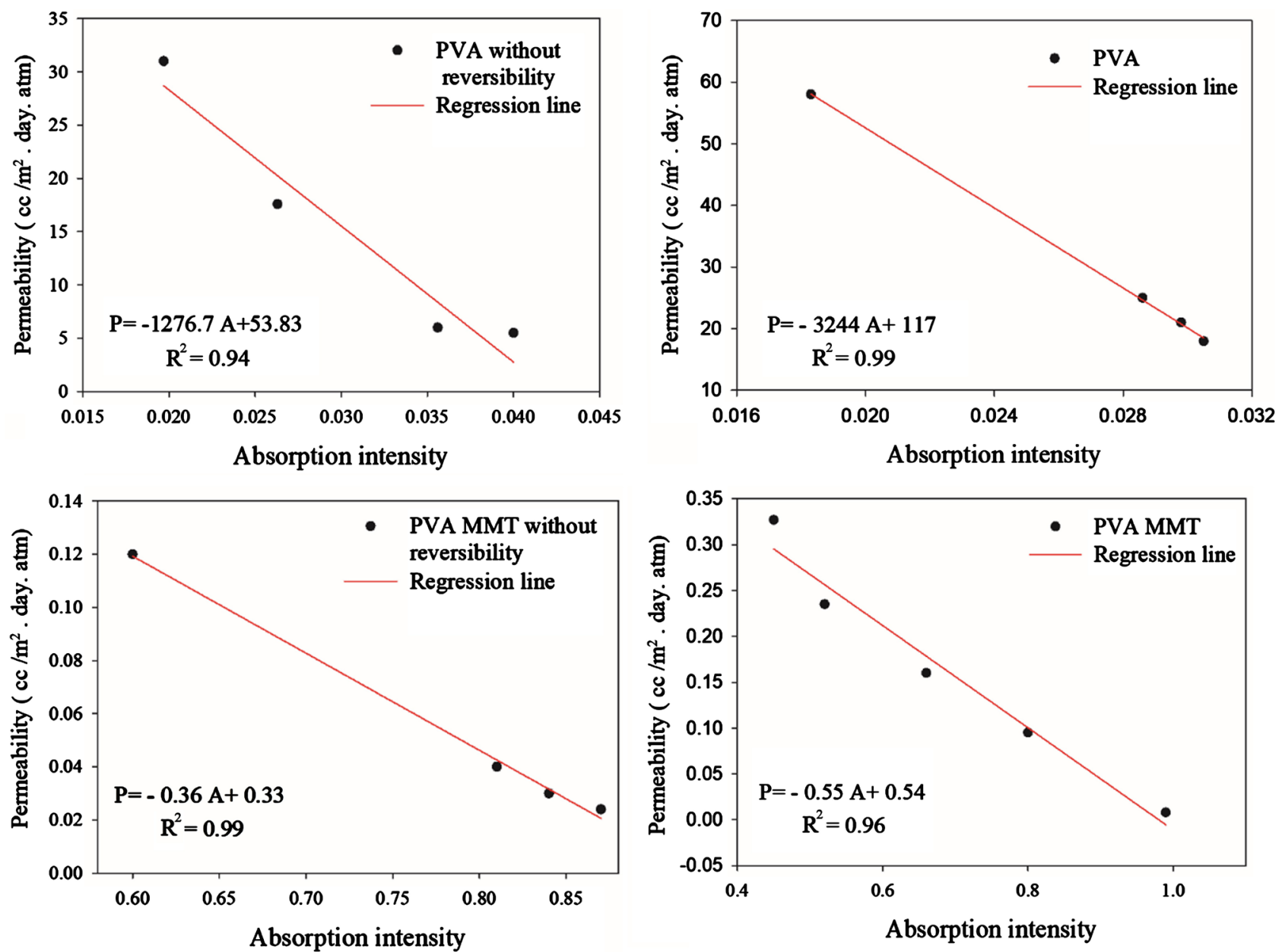

Figure 6. Correlation between the permeability coefficients and FTIR absorption intensity for PVA and PVA-MMT coatings with and without reversibility.

with $\alpha$, the reaction order. As discussed in Section 3.4, permeability is proportional to the FTIR absorption intensity corresponding to the C-O bond. According to Beer Lambert's law, this intensity is linearly proportional to the concentration of the crosslinker in the coating which makes this permeability proportional to concentration. Considering this linearity, the variation of the permeability with the crosslinking time was adopted to study the crosslinking kinetic of PVA. The crosslinking of PVA and PVA-MMT was elaborated with the presence of acid to avoid reversibility. Based on the FTIR correlation, the following reaction between the permeability and the Gly concentration is considered:

$$
P=-a \cdot[\mathrm{Gly}]+b
$$

with $a$ and $b$ constants.

To find the reaction order, $\alpha$, the Gly concentration in Equation (5), is replaced by its expression in function of the permeability, deduced from Equation (6):

$$
-\mathrm{d}\left(\frac{P-b}{a}\right) / \mathrm{d} t=k \cdot\left(\frac{P-b}{a}\right)^{\alpha}
$$


To find the reaction order describing the crosslinking reaction, the linearity of the permeability equation related to each order with time was investigated (Figure 7). For the case of the first reaction order (logarithm of permeability) the linear regression was low $\left(R^{2}=0.7\right)$ compared to second reaction order $\left(R^{2}=\right.$ 0.97), which led to believe that the crosslinking reaction followed a second order reaction based on the Gly.

Integrating Equation (7) while considering a reaction order of two, led to a perfect fit for both types of coating, with Equation (8) that relates permeability values to the crosslinking time (Figure 8).

$$
\frac{1}{P}=k_{\text {app }} \cdot t+\frac{1}{P_{0}}
$$

with $k_{\text {app }}$, the apparent reaction rate constant, as it is the ratio of the reaction rate constant $k$ to the constant $a(k / a)$.

The study of the correlation between permeability and FTIR absorption intensity, showed that the slope constants are not in the same range with a value of 1276.7 for PVA coatings and 0.365 for PVA-MMT coatings. This is mainly due
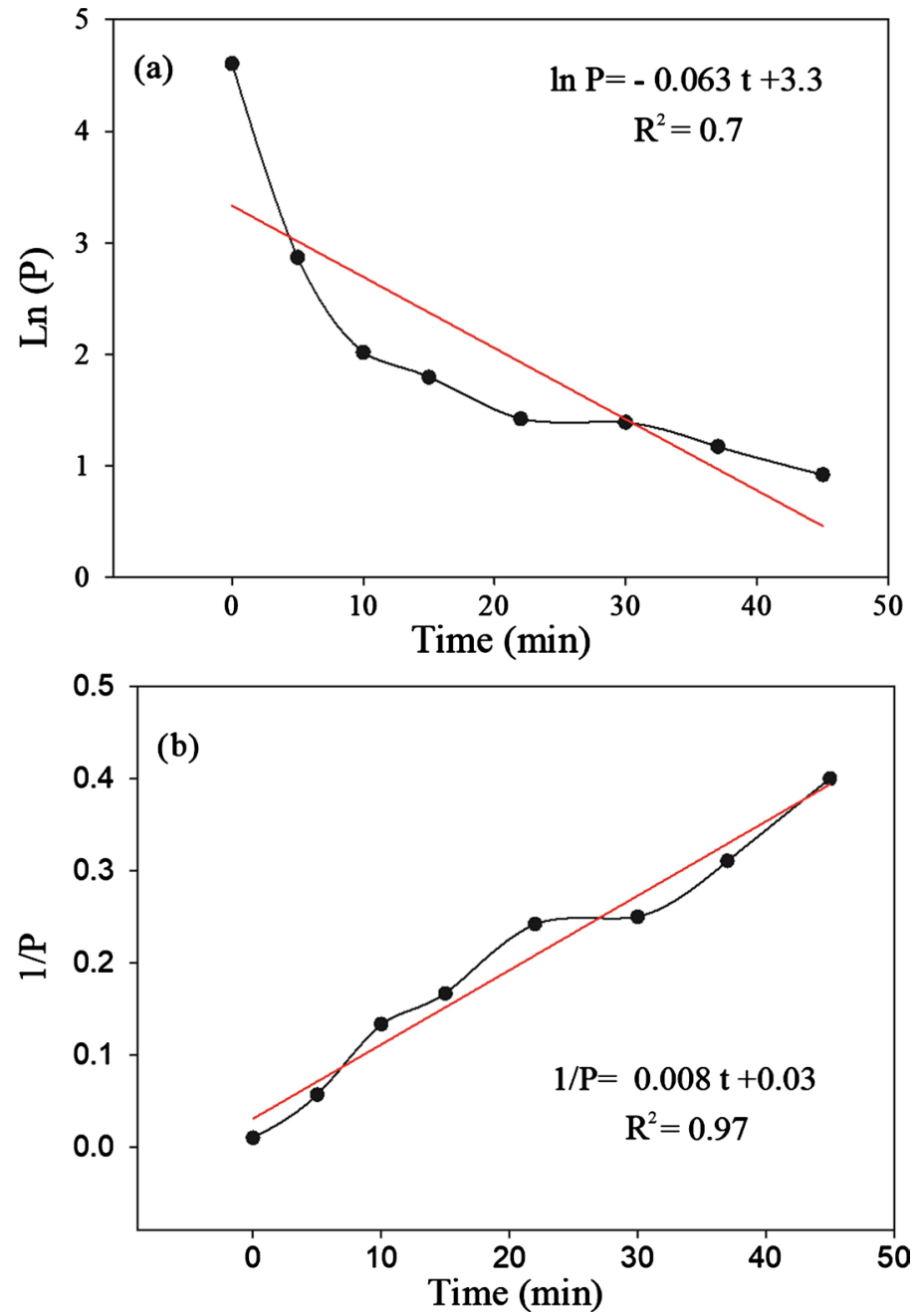

Figure 7. Kinetic evaluation of a crosslinking reaction: (a) Order 1, (b) Order 2. 

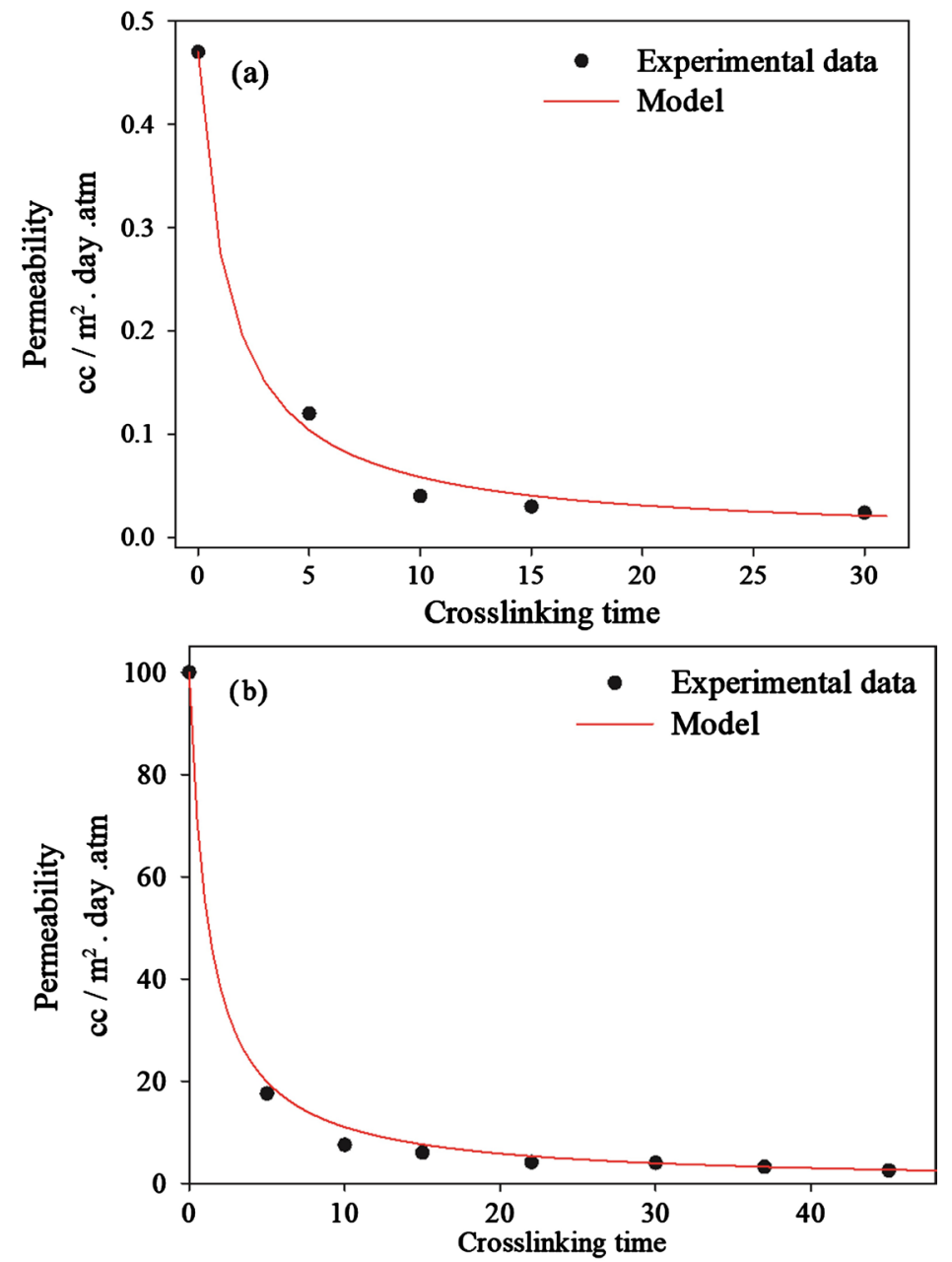

Figure 8. Reaction kinetic of (a) PVA-MMT and (b) PVA coatings without reversibility.

to two reasons. In one side, the interfering of clay peaks with the peak of interest, and in the other side the thicker nanocomposite coatings ( $1 \mu \mathrm{m}$ compare to 0.2 $\mu \mathrm{m}$ for PVA coatings).

According to this second order fitting, the obtained $k_{\text {app }}$ is $1.5 \mathrm{~m}^{2} \cdot$ day $\cdot \mathrm{atm} \cdot \mathrm{cc}^{-1} \cdot \mathrm{min}^{-1}$ for PVA-MMT coating and $0.01 \mathrm{~m}^{2} \cdot$ day $\cdot \mathrm{atm} \cdot \mathrm{cc}^{-1} \cdot \mathrm{min}^{-1}$ for PVA coatings. A straightforward multiplication $a * k_{a p p}$ to obtain $k$, can't be applied in this case, due to the consideration of the constant $a$ in the integration as a slope of the correlation between the permeability and the Gly concentration whereas, only the correlation between the permeability and the FTIR absorption intensity was experimentally obtained.

To be able to compare those values, a correction of the FTIR absorption intensity of PVA-MMT coatings could also be conducted to deduct the effect of clay if needed.

\section{Conclusions}

Crosslinking reaction of PVA with Gly for a LbL deposited film, was studied in this work. It was proven that despite working in heterogeneous systems as LbL, 
the acetal reaction was still reversible. This reaction mechanism was evaluated by the variation of the coated film permeability at various crosslinking times, which allowed to determine the multiple reversibility steps. It was also noticed that the use of fillers in the LbL film did not affect the reaction mechanism since the reversibility was still proven by the permeability and the FTIR studies. To avoid the reversible reaction mechanism, acidic conditions were necessary for the PVA crosslinking.

During the crosslinking study, a strong correlation between the FTIR absorption intensity and the permeability was observed and was interpreted as the result of the proportionality between the solubility coefficient and the crosslinked group concentration.

To complete the study, a kinetic model describing the PVA-Gly crosslinking reaction was performed. As a result, a second order reaction with respect to the Gly was proposed to evaluate the evolution of the permeability with the crosslinking time. The kinetic study was developed assuming that in LbL the polymer is usually in excess compared to the crosslinker, and the linear correlation between the permeability and the concentration of crosslinker, i.e. FTIR measurements. The proposed model with apparent reaction rate constants based on the proposed correlations fitted perfectly the experimental data. It was observed that the use of fillers in the coatings, MMT, increased the apparent reaction rate.

\section{Supporting Information}

TGA thermograms and XRD patterns for different crosslinking times.

\section{Acknowledgements}

The authors are grateful for the financial support of 3SPack NSERC Industrial Research Chair by the Natural Science and Engineering Council of Canada (NSERC) and ProAmpac Inc. Company.

\section{Conflicts of Interest}

The authors declare no conflicts of interest regarding the publication of this paper.

\section{References}

[1] Laufer, G., Kirkland, C., Cain, A.A. and Grunlan, J.C. (2012) Clay-Chitosan Nanobrick Walls: Completely Renewable Gas Barrier and Flame-Retardant Nanocoatings. ACS Applied Materials \& Interfaces, 4, 1643-1649. https://doi.org/10.1021/am2017915

[2] Yagoub, H., Ma, S., Yang, S. and Xu, J. (2014) Preparation and Characterisation of Cellulose Nanocrystals Thin Films Utilising Layer-by-Layer Deposition. Materials Research Innovations, 18, S4-821-S4-824. https://doi.org/10.1179/1432891714Z.000000000801

[3] Luksiene, Z. and Buchovec, I. (2019) Impact of Chlorophyllin-Chitosan Coating and Visible Light on the Microbial Contamination, Shelf Life, Nutritional and Vis- 
ual Quality of Strawberries. Innovative Food Science \& Emerging Technologies, 52, 463-472. https://doi.org/10.1016/j.ifset.2019.02.003

[4] Ploehn, H.J. and Liu, C.Y. (2006) Quantitative Analysis of Montmorillonite Platelet Size by Atomic Force Microscopy. Industrial \& Engineering Chemistry Research, 45, 7025-7034. https://doi.org/10.1021/ie051392r

[5] Ha Thuc, C.N., Cao, H.T., Nguyen, D.M., Tran, M.A., Duclaux, L., Grillet, A.C. and Ha Thuc, H. (2014) Preparation and Characterization of Polyurethane Nanocomposites Using Vietnamese Montmorillonite Modified by Polyol Surfactants. Journal of Nanomaterials, 2014, Article ID: 302735. https://doi.org/10.1155/2014/302735

[6] Cho, C., Wallace, K.L., Hagen, D.A., Stevens, B., Regev, O. and Grunlan, J.C. (2015) Nanobrick Wall Multilayer Thin Films Grown Faster and Stronger Using Electrophoretic Deposition. Nanotechnology, 26, Article ID: 185703.

https://doi.org/10.1088/0957-4484/26/18/185703

[7] Decher, G., Ebler, F., Hong, J.D., Lowack, K., Schmitt, J. and Lvov, Y. (1993) Layer-by-Layer Adsorbed Films of Polyelectrolytes, Proteins or DNA. Polymer Preprints, Division of Polymer Chemistry, American Chemical Society, 34, 745.

[8] Cui, Y.B., Kumar, S., Kona, B.R. and van Houcke, D. (2015) Gas Barrier Properties of Polymer/Clay Nanocomposites. RSC Advances, 5, 63669-63690.

https://doi.org/10.1039/C5RA10333A

[9] Vartiainen, J. and Harlin, A. (2011) Crosslinking as an Efficient Tool for Decreasing Moisture Sensitivity of Biobased Nanocomposite Films. Materials Sciences and Applications, 2, 346-354. https://doi.org/10.4236/msa.2011.25045

[10] Patro, T.U. and Wagner, H.D. (2016) Influence of Graphene Oxide Incorporation and Chemical Cross-Linking on Structure and Mechanical Properties of Layer-by-Layer Assembled Poly(vinyl alcohol)-Laponite Free-Standing Films. Journal of Polymer Science Part B: Polymer Physics, 54, 2377-2387. https://doi.org/10.1002/polb.24226

[11] Zhang, Y., Zhu, P. and Edgren, D. (2009) Crosslinking Reaction of Poly(vinyl alcohol) with Glyoxal. Journal of Polymer Research, 237, 725-730. https://doi.org/10.1007/s10965-009-9362-Z

[12] Figueiredo, K.C.S., Alves, T.L.M. and Borges, C.P. (2009) Poly(vinyl alcohol) Films Crosslinked by Glutaraldehyde under Mild Conditions. Journal of Applied Polymer Science, 111, 3074-3080. https://doi.org/10.1002/app.29263

[13] Rudra, R., Kumar, V. and Kundu, P.P. (2015) Acid Catalysed Cross-Linking of Poly Vinyl Alcohol (PVA) by Glutaraldehyde: Effect of Crosslink Density on the Characteristics of PVA Membranes Used in Single Chambered Microbial Fuel Cells. RSC Advances, 5, 83436-83447. https://doi.org/10.1039/C5RA16068E

[14] Ogata, Y., Okano, M. and Ganke, T. (1956) Kinetics of the Formation of the Formal of Polyvinyl Alcohol. Journal of the American Chemical Society, 78, 2962-2964. https://doi.org/10.1021/ja01594a011

[15] Kormanovskaya, G.N. and Vlodavets, I.N. (1964) Kinetics of the Homogeneous Interaction of Polyvinyl Alcohol with Formaldehyde in Aqueous Solutions. Russian Chemical Bulletin, 13, 1661-1666. https://doi.org/10.1007/BF00849425

[16] Rumyantsev, M., Zelentsov, S.V. and Gushchin, A.V. (2013) Retardation Effect in Acetalization of Poly(vinyl alcohol) with Butyraldehyde. European Polymer Journal, 49, 1698-1706. https://doi.org/10.1016/j.eurpolymj.2013.03.014

[17] Wang, Z.F., Wang, B., Qi, N., Zhang, H.F. and Zhang, L.Q. (2005) Influence of Fillers on Free Volume and Gas Barrier Properties in Styrene-Butadiene Rubber Studied by Positrons. Polymer, 46, 719-724. 
https://doi.org/10.1016/j.polymer.2004.12.002

[18] Choudalakis, G. and Gotsis, A.D. (2009) Permeability of Polymer/Clay Nanocomposites: A Review. European Polymer Journal, 45, 967-984.

https://doi.org/10.1016/j.eurpolymj.2009.01.027 
Supporting Information: Study of the Crosslinking of LbL Nanocomposites with Glyoxal

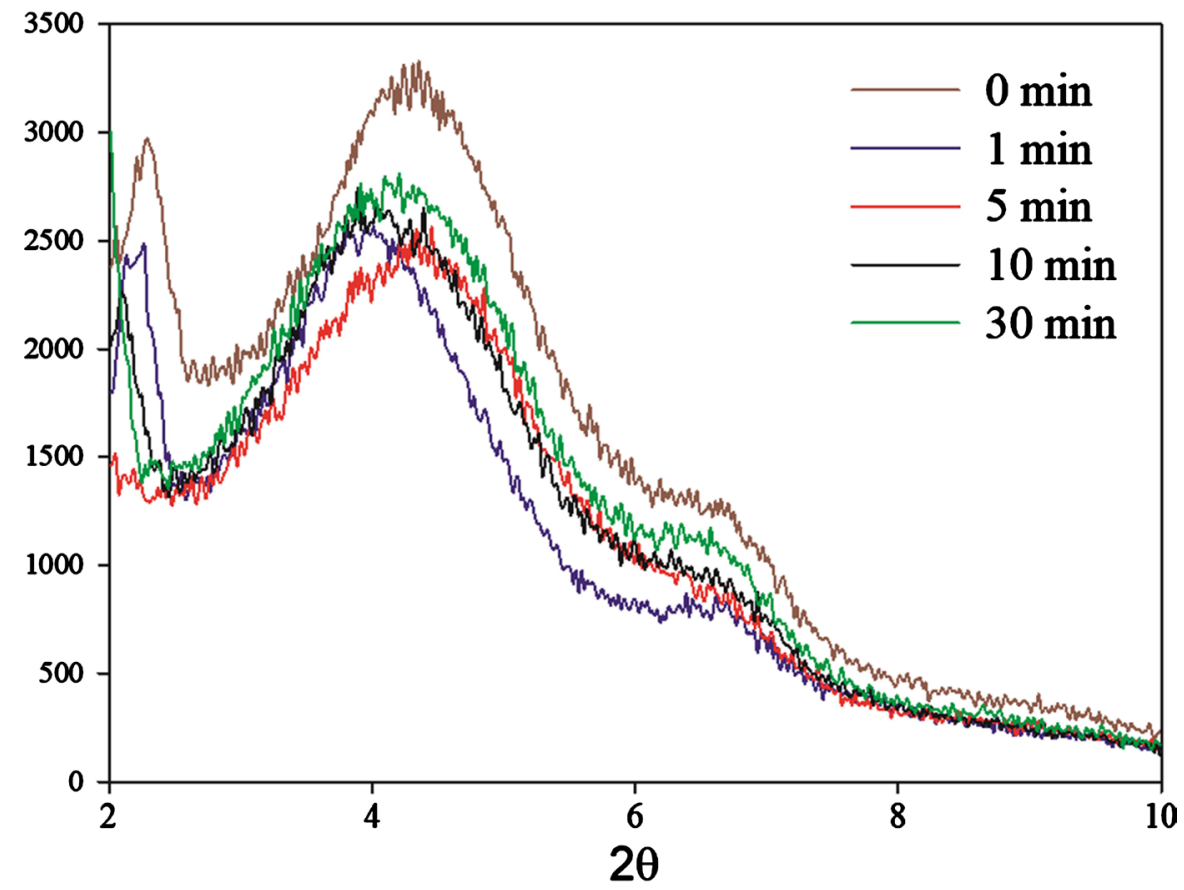

Figure S1. XRDpatterns for different crosslinking times for a PVA-MMT coatings under mild conditions.

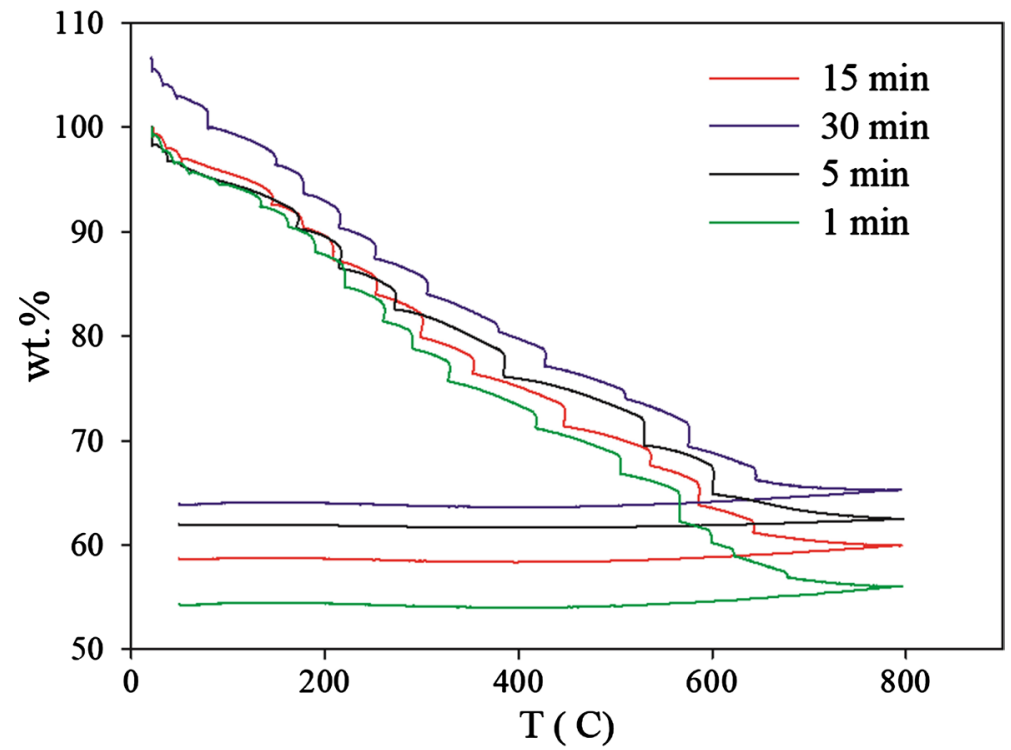

Figure S2. TGA thermograms for crosslinked PVA-MMT coatings under mild condition. 